\title{
Extended Superior Cornu of Thyroid Cartilage Causing Dysphagia and Throat Pain
}

\author{
Piotr Wojtowicz ${ }^{\mathrm{a}}$, Tomasz Szafarowskia, b, Wojciech Kukwa ${ }^{\mathrm{a}}$, Ewa Migacz ${ }^{\mathrm{a}}$, Antoni Krzeski ${ }^{\mathrm{a}}$
}

\begin{abstract}
Superior thyroid cornu syndrome (STCS) is an abnormality of superior cornu of thyroid cartilage which can produce dysphagia, odynophagia or throat pain. The causes of this condition are congenital or acquired. A 51-year-old Caucasian male presented with 4-month history of dysphagia and throat pain. Flexible endoscopy of the larynx revealed an ulceration on the left laryngeal surface of epiglottis. No malignant process was confirmed with histopathological examination, but protruding submucosal mass was detected. CT of the neck showed the ossified and extended left superior thyroid cornu. Surgical procedure was performed to resect the extended cornu. The patient was followed up in 2 weeks and presented no symptoms. STCS is a rare cause of chronic dysphagia or sore throat. Laryngologists should suspect it when malignancy of the larynx is ruled out. Surgery is the only known therapy, but it is not always effective.
\end{abstract}

Keywords: Superior cornu; Thyroid cartilage; Dysphagia; Odynophagia; Throat pain

\section{Introduction}

Ossification of superior thyroid cornu is a rare cause of symptoms such as dysphagia, odynophagia, throat pain or foreign body sensation [1]. Most of the cases concerning the disorders of the thyroid cartilage include a displacement of the superior cornu, either following neck trauma or congenital $[2,3]$. Superior cornu is usually dislocated medially and anterior to its normal position which results in protruding mass toward the lumen of the pharynx. The similar effect is noticed if the superior thyroid cornu is elongated by ossification.

The symptoms of superior thyroid cornu syndrome (STCS)

Manuscript accepted for publication January 14, 2015

aENT Department, Faculty of Medicine and Dentistry, Medical University of Warsaw, Stepinska Street 19/25, 00-739 Warsaw, Poland

${ }^{b}$ Corresponding Author: Tomasz Szafarowski, ENT Department, Faculty of Medicine and Dentistry, Medical University of Warsaw, Stepinska Street 19/25, 00-739 Warsaw, Poland. Email: tomasz.szafarowski@wp.pl

doi: http://dx.doi.org/10.14740/jmc2043w presented in this case report are isolated and do not respond to any conservative treatment.

The thyroid cartilage is not always symmetrical. If a significant asymmetry does not produce any symptoms, it should be left without medical intervention [4].

Flexible endoscopy of the larynx is usually the first step in diagnostic process of STCS, but it may lead to inaccurate diagnosis [5]. Therefore, CT scanning with $3 \mathrm{D}$ reconstruction should be an obligatory part of examination to visualize the thyroid cartilage abnormalities [6]. Although the complete resolution of STCS is ensured by surgical procedure, it is not always the effective treatment and sometimes the symptoms stay unchanged. Despite the symptoms, some patients prefer to avoid surgery, as they are aware that the disease is not a malignant process.

\section{Case Report}

A 51-year-old Caucasian male was presented to ENT department with a 4-month history of sore throat and cervical dysphagia. He was treated conservatively for 4 weeks with Dexaven and antihistamines ordered by ENT doctor with no effect. He had no symptoms of upper respiratory tract infection, hoarseness and dyspnea. He did not recall any history of neck trauma. His medical history included a few episodes of otitis in the childhood. He was not on any medications. A routine ENT examination was unremarkable.

Flexible endoscopy revealed an ulceration on the left laryngeal surface of epiglottis. It suggested a neoplastic lesion of the larynx (Fig. 1B). A direct laryngoscopy under anesthesia was performed with Kleinsasser set in order to obtain samples to histopathological verification. During direct laryngoscopy, the protruding submucosal mass on the left lateral pharyngeal wall was revealed. It irritated the wall of epiglottis and caused the ulcerative lesion. The origin of the indentation was confirmed by touching it with endoscopic forceps. It appeared to be a bone.

CT of the neck with 3D reconstructions was ordered. CT scans demonstrated $20 \mathrm{~mm}$ ossification lengthening left superior thyroid cartilage cornu. The left lateral pharyngeal wall was bulged toward the lumen. The right superior thyroid cornu and the rest of laryngeal cartilages were normal (Fig. 1A). The histopathological examination revealed the ulceration with 


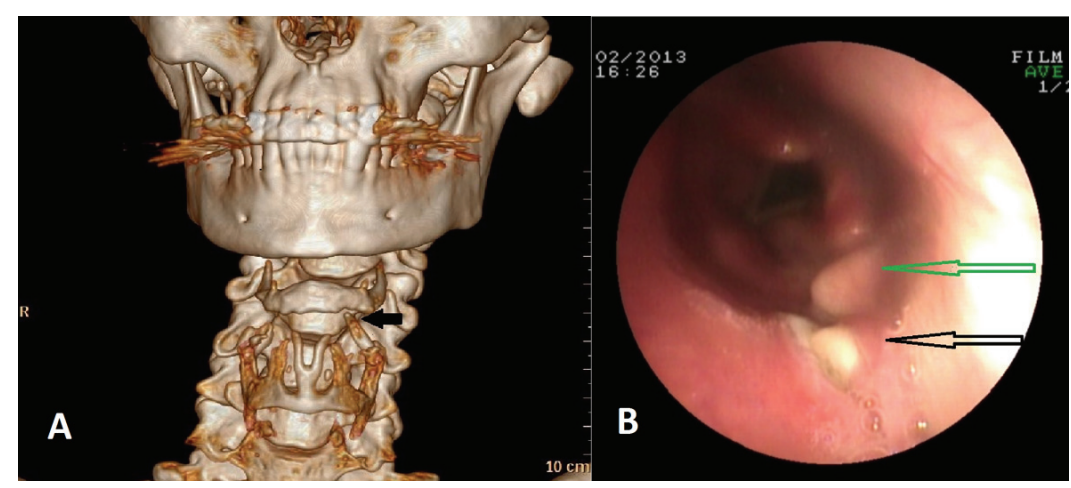

Figure 1. CT scanning of the neck with $3 \mathrm{D}$ reconstruction showing left extended superior thyroid cornu. The arrow points the ossification of superior thyroid cornu. It is also rotated medially. The rest of thyroid cornua are normal (A). Flexible fiberoptic laryngoscopy. Protruding mass on the left pharyngeal wall. The picture shows the submucosal mass protruding towards the pharyngeal lumen (green arrow) and the ulceration on the epiglottis (white arrow) (B).

mucosal and cartilage tissue.

The final diagnosis was an STCS. The patient was informed of a benign nature of the lesion. The option of surgical removal of extended superior thyroid cornu was proposed to him. The consent was obtained. Excision of the affected thyroid cornu was performed with Kleinsasser set under anesthesia. As the pharynx was visualized, the protruding mass was injected with adrenaline. A mucosal incision was made with endoscopic scissors. Mucosa was dissected over the superior thyroid cornu with endoscopic dissector. Thyroid ligament was cut and $21 \mathrm{~mm}$ of cornu was removed with endoscopic scissors. One 4-0 vicryl suture was put on the mucosa (Fig. 2D). Next day patient was able to eat normal diet. Six weeks after surgery, the patient was followed up. He presented complete resolution.

\section{Discussion}

The thyroid cartilage is the largest cartilage in the laryngeal skeleton. Normal anatomy and symmetry of larynx cartilages is responsible for physiological function. Congenital or acquired anatomic variations of the thyroid cartilage can produce various degrees of clinical problems. Current publications present a few cases concerning abnormality in superior cornu of thyroid cartilage. In 1994, Avrahami et al described abnormal location of superior cornu of thyroid cartilage for the first time, which was an effect of the trauma in the past [7]. Smith et al presented 11 patients with asymmetric and damaged superior thyroid cornu which resulted in the posttraumatic odynophagia [8]. Similar cases were reported by

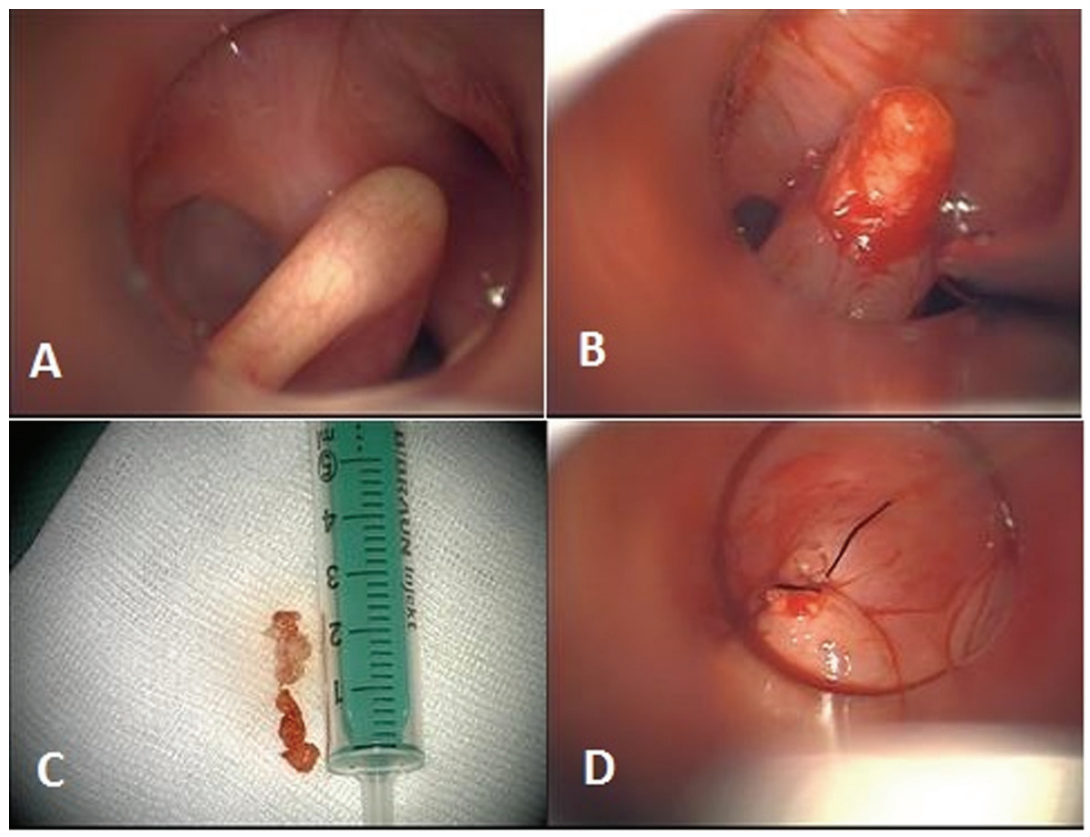

Figure 2. Intraoperative pictures display transoral resection of left superior thyroid cornu. Prominence of cornu (A), dissected mucosa (B), excised ossification of superior thyroid cornu - the length of specimen was about $20 \mathrm{~mm}(C)$, pharyngeal repair (D). 
Nadig et al and Mortensen et al [1, 3]. The neck trauma seems to be the most likely cause of STCS. Strangulation, external compression and endotracheal intubation may produce reposition of superior cornu which is bulging toward laryngeal lumen [7]. Clinically symptomatic STCS may develop even a few years after the trauma. The congenital defect of superior cornu, including inferior constrictor of fourth branchial arch mal development, is also suggested to be the cause of STCS. However, it is still not confirmed. Ossification of thyroid cartilage starts at the age of $20-23$ years at the inferior margin. Therefore, only dislocation of superior cornu may occur in this situation. Superior thyroid cornu which is bulging towards lumen produces many symptoms ranging from globus sensation to odynophagia. The differential diagnosis should be at first based on excluding malignancy, then reflux disease, allergy and neurological dysfunction.

Flexible endoscopy is a very useful examination, but may be not sufficient to confirm the diagnosis. The most important is to examine the abnormality in superior thyroid cornu with CT scanning with $3 \mathrm{D}$ reconstructions.

If benign lesion is confirmed, the therapeutic options should be presented to the patient. Surgery procedure is the only known therapy. Although surgical procedure gives almost always relief in symptoms, some patients refuse it. They are satisfied with the explanation of the benign nature of the disease. In this situation, an annually follow-up is recommended.

Although the STCS is a rare disease, the laryngologists should be aware of it, when isolated symptoms of sore throat, dysphagia, odynophagia or foreign body sensation are presented.

\section{Abbreviations}

STCS: superior thyroid cornu syndrome

\section{Competing Interests}

The authors declare that they have no competing interests.

\section{References}

1. Mortensen M, Ivey CM, Iida M, Woo P. Superior thyroid cornu syndrome: an unusual cause of cervical dysphagia. Ann Otol Rhinol Laryngol. 2009;118(12):833-838.

2. Lin D, Fischbein N, Eisele DW. Odynophagia secondary to variant thyroid cartilage anatomy. Dysphagia. 2005;20(3):232-234

3. Nadig SK, Uppal S, Back GW, Coatesworth AP, Grace AR. Foreign body sensation in the throat due to displacement of the superior cornu of the thyroid cartilage: two cases and a literature review. J Laryngol Otol. 2006;120(7):608-609.

4. Turkmen S, Cansu A, Turedi S, Eryigit U, Sahin A, Gunduz A, Shavit I. Age-dependent structural and radiological changes in the larynx. Clin Radiol. 2012;67(11):e2226.

5. Hajiioannou JK, Florou V, Kousoulis P. Superior thyroid cornu anatomical variation causing globus pharyngeous and Dysphagia. Case Rep Med. 2010;2010:142928.

6. Browning ST, Whittet HB. A new and clinically symptomatic variant of thyroid cartilage anatomy. Clin Anat. 2000;13(4):294-297.

7. Avrahami E, Harel M, Englender M. CT evaluation of displaced superior cornu of ossified thyroid cartilage. Clin Radiol. 1994;49(10):683-685.

8. Smith ME, Berke GS, Gray SD, Dove H, Harnsberger R. Clicking in the throat: cinematic fiction or surgical fact? Arch Otolaryngol Head Neck Surg. 2001;127(9):11291131. 